\title{
Réponses et cadres conceptuels des cliniciens enseignants lors de discussions de cas avec des internes en psychiatrie
}

\author{
Responses and conceptual frameworks of preceptors' feedback during case \\ discussions with residents in psychiatry
}

\author{
Luc CÔTÉ ${ }^{1}$ et Nathalie GINGRAS ${ }^{2}$ \\ 1 Département de médecine familiale et de médecine d'urgence, Faculté de médecine, Université Laval, Québec, Canada \\ 2 Département de psychiatrie et neurosciences, Faculté de médecine, Université Laval, Québec, Canada
}

Manuscrit soumis à la rédaction le 25 février 2013 ; commentaires éditoriaux formulés aux auteurs le 19 mars 2103 ; accepté pour publication le 4 juillet 2013

\begin{abstract}
Mots-clés
Supervision clinique ;

internat ;

rétroaction ;

cliniciens enseignants

Résumé - Contexte et problématique : La rétroaction constructive reçue en cours de formation est essentielle au développement de l'expertise. Or, la majorité des études ou des guides se rapportant à la supervision mettent l'accent sur « comment » échanger la rétroaction et non sur son contenu. De même, aucune étude n'a porté sur les bases ou les cadres conceptuels soustendant le contenu de la rétroaction. Objectifs : Décrire les réponses des cliniciens enseignants aux demandes pédagogiques formulées par des internes (ou résidents) en psychiatrie lors de la discussion de cas ; 2) Répertorier les bases conceptuelles sur lesquelles reposent les réponses ; 3) Explorer s'il y a des différences entre les réponses et les bases conceptuelles selon l'expérience des cliniciens. Matériel et méthodes : Une étude qualitative a été réalisée auprès de 21 cliniciens enseignants en psychiatrie de l'Université Laval. Lors d'une entrevue individuelle, ils ont d'abord répondu à quatre vignettes écrites de supervision dans lesquelles l'interne présentait explicitement une demande pédagogique. Par la suite, ils devaient expliquer le rationnel de leurs réponses. Une analyse thématique de contenu des verbatim a été faite, avec triangulation des chercheurs, suivie d'un calcul des occurrences. Résultats : Les vignettes ont généré 450 réponses se rapportant à 85 thèmes distincts associés à quatre compétences professionnelles. Plus de $60 \%$ des justifications reposaient sur des cadres conceptuels. Des différences ont aussi été observées selon le niveau d'expérience des cliniciens enseignants. Conclusion : Cette étude aura permis de documenter la richesse des réponses des cliniciens enseignants ainsi que les bases conceptuelles sur lesquelles reposent ces réponses. Dans une optique de valorisation et d'application des connaissances, les résultats serviront à l'élaboration d'activités de formation pédagogique pour la supervision en contexte clinique.
\end{abstract}




\begin{abstract}
Keywords
Clinical supervision; residency; feedback; clinical teacher

Abstract - Background: The development of clinical expertise is highly influenced by the quality of constructive feedback provided during clinical training. However, much of the research and literature on clinical supervision focuses on "how" to provide feedback and much less on the content of feedback. Additionally, there are no studies that examine the conceptual frameworks that guide the content of feedback. Purpose: 1) Describe the content of the feedback provided by clinical supervisors (or preceptors) to resident learning requests; 2) Identify the conceptual frameworks reported by clinical supervisors as underlying their feedback; 3) Explore any differences in conceptual frameworks and the content of feedback based on clinical supervisor experience. Methods: Twenty-one clinical supervisors in psychiatry from Laval University participated in individual qualitative interviews. During the interviews supervisors were presented with four written vignettes, each depicting a resident seeking help in a specific clinical case. Preceptors were first asked how they would respond in each situation, and then what the rationale or bases for their responses were. We analyzed the data using thematic content analysis and frequency counts. Several researchers were involved to triangulate analysis. Results: The vignettes generated 450 responses representing 85 distinct themes related to four professional competencies. More than $60 \%$ of the responses were informed by an underlying conceptual framework. There were differences in the type and complexity of conceptual framework used, and the nature of response according to the level of supervisor experience. Conclusion: Findings from the study highlight the diversity of feedback provided, and the range of frameworks used by preceptors during clinical supervision. The findings have important implications for teaching and faculty development in the context of clinical supervision.
\end{abstract}

\section{Introduction}

\section{Problématique}

En éducation médicale, il est reconnu que la formation clinique est une étape charnière dans le développement des compétences et de l'identité professionnelle du médecin et que la supervision clinique est au cœur de cet apprentissage. En effet, pendant les stages, les externes et les internes (ou résidents) sont en contact avec les réalités de la profession, sont exposés à différents modèles de pratique et à différents styles de superviseurs, en même temps qu'ils développent quotidiennement des apprentissages relatifs à l'exercice de leur profession ${ }^{[1-5]}$. En accord avec la théorie de l'apprentissage contextualisé, l'intégration à des communautés de pratique, notamment en stage, crée les conditions les plus favorables pour que les futurs médecins adoptent progressivement la culture et les compétences de leur profession ${ }^{[6]}$

La présence des cliniciens enseignants (ou superviseurs) est essentielle au développement de compétences professionnelles. Ceci est d'autant plus vrai si ceux-ci sont perçus comme des modèles de rôle positifs par les étudiants. Selon la perspective de la pratique délibérée et intentionnelle -deliberate practice- ${ }^{[7,8]}$, qui est l'une des théories qui rend compte du développement de l'expertise, celui-ci repose sur une pratique variée, intense et délibérée dans laquelle l'étudiant se pose des défis de plus en plus importants au plan de l'apprentissage. De plus, l'expertise s'acquiert lors de la rétroaction reçue en cours de formation, notamment lorsque les apprentissages sont plus difficiles. Ainsi, les superviseurs stimulent les étudiants à se dépasser et les aident notamment à identifier les difficultés qu'ils rencontrent ainsi que diverses stratégies pour les résoudre. Cette démarche vient non seulement structurer mais consolider les apprentissages.

Les cliniciens enseignants doivent relever plusieurs défis afin de favoriser le développement des compétences professionnelles des internes $\mathrm{s}$ lors de la discussion des cas. Contraints par le temps, puisqu'ils ne disposent généralement que de cinq à neuf minutes pour la discussion en contexte ambulatoire ${ }^{[9,10]}$, ils doivent gérer le cas, évaluer les besoins d'apprentissage des étudiants en fonction du cas et échanger une 
rétroaction pour consolider les acquis et corriger les erreurs, le cas échéant. L'importance de ce double raisonnement, clinique et pédagogique, du superviseur a récemment été rappelée par Jouquan ${ }^{[11]}$.

Les premiers modèles de rétroaction pédagogique, fortement basés sur le paradigme behavioriste de l'apprentissage, stipulaient que les interventions des superviseurs devaient être strictement centrées sur les comportements observés, alors que les modèles plus récents, tel que celui du compagnonnage cognitif $^{[12]}$, s'adossent au paradigme socio-constructiviste de l'apprentissage. Une telle orientation nécessite d'explorer pendant la rétroaction autant le résultat de l'action professionnelle qui est supervisée que le processus ayant mené l'étudiant à cette action. Pour le superviseur, la rétroaction comporte donc deux dimensions complémentaires ${ }^{[13,14]}$ : a) le contenu discuté, idéalement en fonction des besoins d'apprentissage de l'étudiant -le quoi-, qui a trait par exemple à la clarification et la discussion des questions de l'étudiant en relation avec la démarche clinique, la relation avec le patient, l'organisation de l'entrevue, etc. ; b) le processus par lequel la rétroaction est faite -le comment-, tel que le proposent par exemple certains formats codifiés de supervision, comme celui de « la minute du superviseur »-One Minute Preceptor ${ }^{-}{ }^{[15]}$ ou encore celui désigné par l'acronyme mnémonique SNAPPS ${ }^{[16]}$ -pour : Summarize ; Narrow ; Analyze ; Probe ; Plan ; Select.

Bien que plusieurs publications en éducation médicale portent sur la rétroaction, la majorité des études ou des guides proposés met l'accent sur « comment» échanger la rétroaction et non sur son contenu $^{[1,4]}$. Une des stratégies pédagogiques pour identifier les besoins d'apprentissage d'un étudiant lorsqu'il présente un cas et, ainsi, développer sa pratique réflexive en fonction de ses besoins, consiste à lui demander d'exprimer ses incertitudes ou ses questions en relation avec le cas ${ }^{[17-19]}$. Si elle est bien faite, tant au niveau du contenu que du processus, la rétroaction fournie par le superviseur à partir de ces questions ou incertitudes a une double fonction : elle stimule la pratique réflexive chez l'étudiant tout en lui fournissant une évaluation externe sur ses habiletés cognitives, métacognitives et relationnelles ${ }^{[20]}$. De plus, l'impact de cette rétroaction peut être significativement amélioré si la relation superviseur-apprenant favorise chez l'étudiant la position d'apprentissage plutôt que celle d'évaluation ${ }^{[21]}$.

Le contenu de la rétroaction peut inclure une variété de sujets. Même si certains sujets sont inévitables (la discussion du cas clinique), le contenu de ce qui est dit peut varier selon, par exemple, la nature du cas, la nature des questions ou des incertitudes de l'étudiant, le confort du superviseur avec certains sujets, le contexte de la supervision et le temps disponible. Bien que les influences soient multifactorielles, un des facteurs capables d'orienter le contenu de la rétroaction du superviseur est le cadre conceptuel ou théorique, explicite ou implicite, qu'il a en mémoire en relation avec la question posée ou le sujet discuté. Un cadre conceptuel est une façon de se représenter les situations ; il s'agit en fait d'une grille de lecture et d'analyse des phénomènes complexes. Selon Bordage ${ }^{[22]}$, les théories, les meilleures pratiques et les modèles ou approches constituent les principales catégories de cadres conceptuels. Les théories (T), par exemple, la théorie du développement de l'expertise, consistent en un ensemble de propositions ou de principes bien organisés. Ils sont issus d'expériences et ont une valeur prédictive. Les meilleures pratiques (MP) sont des recommandations de pratiques professionnelles (guidelines) proposées par des groupes d'experts. Par exemple, il est recommandé d'implanter progressivement des changements d'habitudes de vie chez un patient. Quant aux modèles ou approches $(\mathrm{M})$, ils découlent des théories. Ils sont des représentations plus ou moins complexes de démarches servant de référence ; l'approche clinique dite « centrée sur le patient »-patient centered careen est un exemple. Enfin, à un niveau meta-épistémologique, les paradigmes, tels que les paradigmes positiviste et constructiviste, constituent également des cadres théoriques mobilisables par les cliniciens enseignants.

En 2008, Côté et Bordage ont initié un programme de recherche sur le contenu des réponses des superviseurs à des demandes formulées par les internes lors de la discussion de cas et sur les justifications sous-tendant ces réponses. Une première étude réalisée en médecine familiale et en médecine interne à l'Université Laval ${ }^{[23]}$ montre que les cliniciens enseignants donnent des réponses variées et qu'ils 
Tableau I. Provenance et caractéristiques des participants.

\begin{tabular}{|l|l|l|l|}
\hline \multicolumn{1}{|c|}{$\begin{array}{c}\text { Pédopsychiatrie } \\
(\boldsymbol{n}=\mathbf{7})\end{array}$} & \multicolumn{1}{|c|}{$\begin{array}{c}\text { Psychiatrie adulte } \\
(\boldsymbol{n = 1 2})\end{array}$} & \multicolumn{1}{|c|}{$\begin{array}{c}\text { Gérontopsychiatrie } \\
(\boldsymbol{n = 2 )}\end{array}$} & \multicolumn{1}{c|}{$\begin{array}{c}\text { Total } \\
(\boldsymbol{n}=\mathbf{2 1})\end{array}$} \\
\hline Hommes $: 5$ & Hommes $: 5$ & Homme $: 1$ & Hommes $: 11$ \\
Femmes $: 2$ & Femmes $: 7$ & Femme $: 1$ & Femmes $: 10$ \\
Expérience $: 5-38$ ans & Expérience $: 4-41$ ans \\
(moyenne $: 18,7$ ans) & Expérience $: 16-26$ ans \\
$\leq 5$ ans $: 3$ & $\leq 13$ ans $: 5$ & Expérience $: 4-41$ ans \\
(moyenne $: 21$ ans) & & $4-13$ ans $: 8$ \\
$\geq 24$ ans $: 4$ & $\geq 17$ ans $: 7$ & & $17-41$ ans $: 13$ \\
\hline
\end{tabular}

sont en mesure d'identifier des cadres conceptuels sur lesquels reposent ces réponses. Le présent projet auprès des psychiatres s'inscrit dans le cadre de ce programme et a été réalisé en même temps que le précédent projet, d'abord dans une optique de comparer les réponses et les cadres conceptuels avec une population différente de cliniciens enseignants, soit des psychiatres provenant de la même université. De plus, la présente étude constitue une forme de triangulation qui donne du poids à la crédibilité et à la transférabilité des données issues de ces deux études.

\section{Questions de recherche}

L'étude visait à répondre aux trois questions de recherche suivantes :

Question 1: Quelles sont les réponses des cliniciens enseignants en psychiatrie aux demandes pédagogiques formulées par les internes lors de la présentation de cas?

Question 2: Quelles sont les justifications ou bases conceptuelles ou théoriques sur lesquelles reposent les réponses des cliniciens enseignants ?

Question 3 : Est-ce que les réponses et les justifications, ou bases conceptuelles ou théoriques, sont similaires selon qu'elles sont formulées respectivement par des cliniciens enseignants moins expérimentés ou plus expérimentés ?

\section{Méthodes}

\section{Participants et recrutement}

Entre 2009 et 2011, nous avons réalisé une étude qualitative, à visée descriptive. Pendant l'automne 2009, nous avons sollicité, au moyen d'une lettre personnalisée, l'ensemble des pédopsychiatres, psychiatres généraux et gérontopsychiatres associés au réseau d'enseignement clinique de l'Université Laval afin de les inviter à participer à l'étude. Un formulaire d'information et de consentement accompagnait la lettre d'invitation. Dans ce formulaire, on indiquait que ce projet avait été approuvé par le comité d'éthique de la recherche de l'Université Laval. Pour participer, les psychiatres devaient répondre aux critères d'éligibilité suivants : 1) exercer dans le réseau de l'Université Laval dans l'une ou l'autre des spécialités suivantes : pédopsychiatrie, psychiatrie générale et gérontopsychiatrie ;2) superviser des internes en psychiatrie de façon régulière ; 3 ) accepter de participer à l'étude et signer le formulaire de consentement de participation.

Nous avons inclus les 21 psychiatres ayant répondu positivement à notre invitation et correspondant aux critères d'éligibilité (Tableau I). Cet échantillon était constitué d'hommes et de femmes provenant de différentes spécialités en psychiatrie et ayant des années d'expérience variables. De plus, nous avons atteint la saturation des données à la $15^{\text {ème }}$ entrevue. Ainsi, nous avons respecté les critères de scientificité en recherche qualitative que sont la diversité des participants et la saturation des données ${ }^{[24,25]}$.

\section{Recueil des données}

Lors d'une entrevue individuelle en face-à-face, les participants devaient répondre à quatre courtes vignettes écrites de présentation de cas, mettant explicitement en évidence une demande pédagogique 
associée au cas. Chaque demande se référait à une des compétences identifiées dans le cadre de compétences CanMEDS, qui constitue la référence pour la formation des internes canadiens ${ }^{[26]}$ (cf. Annexe 1) :

- vignette 1 : l'organisation de l'entrevue (compétence « gestion»)

- vignette $2:$ le raisonnement clinique (compétence « expertise médicale »)

- vignette 3 : la relation médecin-patient (compétence « communication »)

- vignette 4 : l'utilisation des ressources éducatives (compétence « érudition »).

Ces demandes étaient parfois présentées de manière générale (par exemple : «Je fais souvent des consultations trop longues et j'aimerais être plus efficace ») et d'autres en termes plus spécifiques (par exemple : à la suite d'une présentation d'un patient ayant un trouble obsessionnel compulsif, l'interne demande: «Qu'est-ce que je pourrais lire sur ce sujet afin d'améliorer mes connaissances, sans passer des heures et me perdre dans mes lectures ?»). Ces vignettes ont été adaptées à la psychiatrie à partir de celles élaborées et utilisées dans le cadre de l'étude de Côté et Bordage ${ }^{[23]}$. Elles ont été pré-testées auprès de la directrice du programme de résidence en psychiatrie de l'Université Laval, ainsi qu'auprès d'une résidente senior de ce programme. Des modifications mineures ont été apportées afin d'en améliorer la clarté.

Après avoir présenté la vignette écrite au participant, l'interviewer utilisait la technique du raisonnement à voix haute (think aloud) ${ }^{[27]}$, en demandant au superviseur : «Que répondriez-vous spontanément à cet interne dans cette situation? » Afin d'éviter l'effet de séquence, les mêmes vignettes ont été présentées aux participants mais selon un ordre déterminé de manière aléatoire. Un maximum de cinq minutes était alloué pour répondre à chacune des vignettes afin de reproduire le contexte de la « vraie » vie où le superviseur est souvent limité par le temps. Lors de l'entretien, l'interviewer notait les réponses du participant. Une fois les réponses complétées pour chacune des vignettes, l'interviewer revenait sur les éléments essentiels des réponses du participant à chacune des vignettes en utilisant la technique du rappel stimulé ${ }^{[28]}$ (stimulated recall). Il devait résumer les principales réponses du superviseur puis lui demander : « Pouvezvous m'expliquer le rationnel de vos réponses, les bases ou les fondements sur lesquels elles reposent? ».

\section{Analyse des données}

Les entrevues ont été enregistrées en audio et transcrites intégralement. L'analyse de contenu thématique des verbatims s'est faite selon la méthode décrite par L'Écuyer ${ }^{[29]}$ à partir de catégories émergeantes. Par un processus itératif de triangulation, les chercheurs ont mis en commun leurs codifications respectives pour enrichir le cahier de codage (deux chercheurs au moins ont analysé les données de chaque verbatim). Lorsque le sens d'une unité d'information était compris différemment par l'un ou l'autre des membres de l'équipe, la codification finale se faisait par consensus. Les membres ont révisé périodiquement le travail d'analyse afin de s'assurer de la pertinence et de l'exhaustivité des catégories. Une fois l'analyse inductive complétée, nous avons quantifié les occurrences, uniquement dans le but d'identifier les réponses et les cadres conceptuels les plus fréquemment observés. Les deux auteurs de l'article ont fait la synthèse finale de l'analyse et rédigé le rapport de recherche.

\section{Résultats}

Les principaux résultats seront présentés en fonction de chacune des questions de recherche.

«Quelles sont les réponses des cliniciens enseignants en psychiatrie aux demandes pédagogiques formulées par les internes lors de la présentation de cas? »

Au total, les cliniciens ont généré 450 réponses, soit une moyenne de 5,4 réponses par clinicien et par vignette (si le clinicien répétait la même réponse dans la vignette, elle n'était comptée qu'une seule fois). Les réponses les plus fréquentes, toutes vignettes confondues, et les réponses les plus fréquentes par vignette sont présentées respectivement dans les tableaux II et III. 
Tableau II. Réponses des cliniciens-enseignants les plus fréquentes, avec exemples verbatim, toutes vignettes confondues.

\begin{tabular}{|c|c|}
\hline Type de réponse (et fréquence) & Exemples verbatim \\
\hline $\begin{array}{l}\text { Suggérer des lectures ou des ressources documentaires } \\
\text { internet (19) }\end{array}$ & $\begin{array}{l}\text { Je lui dirais d'aller lire dans un textbook comme } \\
\text { Kaplan, par exemple. }\end{array}$ \\
\hline $\begin{array}{l}\text { Donner des conseils pour favoriser l'alliance } \\
\text { thérapeutique (18) }\end{array}$ & $\begin{array}{l}\text { C'est important de manifester son empathie au patient } \\
\text { qui vit une situation difficile car cela aide à développer } \\
\text { l'alliance médecin-patient. }\end{array}$ \\
\hline $\begin{array}{l}\text { Poser un diagnostic pédagogique provisoire ou définitif } \\
\text { (17) }\end{array}$ & $\begin{array}{l}\text { Ici, on est en présence d'un résident qui a un problème } \\
\text { de raisonnement clinique car il ne réussit pas à se faire } \\
\text { une idée diagnostique du problème. }\end{array}$ \\
\hline $\begin{array}{l}\text { Donner des moyens concrets pour trouver } \\
\text { les diagnostics différentiel et principal (17) }\end{array}$ & $\begin{array}{l}\text { Je lui demanderais de faire un tableau et de mettre ce } \\
\text { qui correspond avec tel diagnostic et tel autre. }\end{array}$ \\
\hline Donner des conseils sur les techniques d'entrevue (15) & $\begin{array}{l}\text { Parfois on n'ose pas trop interrompre } \\
\text { le patient, mais avec ce genre de patient, il faut } \\
\text { le faire, sinon on n'arrive pas à gérer notre } \\
\text { temps, on n'arrive pas à avoir des réponses à } \\
\text { nos questions. }\end{array}$ \\
\hline $\begin{array}{l}\text { Souligner l'importance d'explorer le lien thérapeutique } \\
\text { du patient avec son médecin antérieur (14) }\end{array}$ & $\begin{array}{l}\text { Ensuite, je lui parlerais de l'importance } \\
\text { justement de faire parler le patient sur le } \\
\text { changement de psychiatre traitant, sur son lien } \\
\text { avec le psychiatre précédent car c'est } \\
\text { important pour les patients. }\end{array}$ \\
\hline Explorer le vécu du résident : problématique vécue (13) & $\begin{array}{l}\text { C'est de voir dans cette situation-là ce qui inquiète le } \\
\text { résident au point de devoir venir m'en parler tout de } \\
\text { suite ; il y a certainement quelque chose qui l'inquiète } \\
\text { chez ce patient-là. }\end{array}$ \\
\hline $\begin{array}{l}\text { Exprimer au résident son soutien : problématique vécue } \\
\text { (13) }\end{array}$ & $\begin{array}{l}\text { Je dédramatiserais la situation en disant que ce n'est } \\
\text { pas facile de faire une entrevue avec un patient } \\
\text { circonstanciel et qu'on va essayer de trouver des } \\
\text { moyens pour l'aider à gérer la situation. }\end{array}$ \\
\hline $\begin{array}{l}\text { Aider le résident à préciser son diagnostic pédagogique } \\
\text { (13) }\end{array}$ & $\begin{array}{l}\text { Le résident dit que son problème, c'est qu'il est } \\
\text { toujours en retard... Je regarderais comment ça } \\
\text { fonctionne une journée pour lui, comment il s'organise, } \\
\text { comment il gère son temps afin de bien saisir où est le } \\
\text { problème. }\end{array}$ \\
\hline
\end{tabular}


Tableau III. Réponses des cliniciens-enseignants les plus fréquentes par vignette.

Vignette 1: «J'aimerais bien être plus efficace » (gestion d'entrevue)

Donner des conseils sur les techniques d'entrevues (ex. : doser les questions ouvertes

et fermées ; reformuler)

Donner des conseils sur la gestion d'un cas (ex. : établir un contrat thérapeutique)

Aider le résident à préciser son diagnostic pédagogique (ex. : comment il explique sa difficulté)

Donner des conseils sur la gestion de l'horaire (ex. : diviser l'entrevue)

Vignette 2 : «Je n'arrive pas à me faire une idée » (raisonnement clinique)

Demander au résident des précisions sur la problématique clinique, faire raconter l'histoire par le résident

Vérifier les connaissances factuelles du résident

Donner des conseils sur l'approche de traitement

Demander au résident de comparer/contraster les diagnostics

Vignette 3 : «Avez-vous des trucs à me donner ?» (relation médecin-patient)

Donner des conseils pour favoriser l'alliance thérapeutique

Souligner l'importance d'explorer le lien thérapeutique patient-médecin antérieur

Souligner l'importance d'établir une bonne alliance thérapeutique comme condition d'une bonne relation patient-médecin

Aider le résident à approfondir la compréhension de ce qui se passe avec le patient

Vignette 4 : «Qu'est-ce que je pourrais lire ?» (choix de ressources éducatives)

Suggérer des lectures ou ressources internet

Proposer un moyen pour chercher l'information (ex. : interroger la base de données Medline ensemble)

Explorer les ressources d'information utilisées

Explorer la démarche de recherche d'information du résident

* Nombre de précepteurs ayant donné cette réponse. Si le précepteur mentionnait cette réponse à plus d'une reprise dans une même vignette, la réponse n'était comptée qu'une seule fois.

Sur les 450 réponses générées, 324 (72\%) se rapportaient à une seule vignette ou compétence spécifique, alors que $126(28 \%)$ se rapportaient à au moins deux vignettes. Les réponses associées à l'exploration du vécu du résident face à la problématique discutée, ainsi que l'utilisation de stratégies pédagogiques (par exemple : demander au résident de clarifier la nature de ses difficultés) n'étaient pas spécifiques à une vignette en particulier.

Par ailleurs, à partir des 450 réponses, nous avons identifié 85 thèmes distincts se rapportant aux compétences «érudition », « expertise médicale », « communication » et « gestion ». Ces thèmes ont été regroupés sous 17 catégories. La liste détaillée des réponses par catégories est présentée dans l'annexe 2.

\section{«Quelles sont les justifications ou bases concep- tuelles ou théoriques sur lesquelles reposent les réponses des cliniciens enseignants ?»}

Au total, les cliniciens ont produit 75 justifications à leurs réponses. Quarante six (61\%) justifications se rapportaient à des cadres conceptuels, 
respectivement à 12 modèles ou approches distinctes, cinq meilleures pratiques distinctes, une théorie et une typologie nosographique. Les 29 autres justifications $(39 \%)$ se rapportaient à la formation que les cliniciens avaient eux-mêmes reçue pendant leur formation ou à l'expérience acquise depuis l'entrée en pratique, sans que des cadres conceptuels soient explicitement exprimés.

La liste des cadres conceptuels distincts explicitement rapportés par vignette par les superviseurs (et non déduits par les chercheurs) est présentée dans le tableau IV.

« Est-ce que les réponses et les justifications ou bases conceptuelles ou théoriques sont similaires entre les cliniciens enseignants moins expérimentés comparé à ceux plus expérimentés ? »

Pour répondre à cette question, nous avons constitué, à titre exploratoire, deux groupes les plus égaux possibles pour les fins de l'analyse: les «moins » expérimentés ( $n=8 ; 4-13$ années) et les « plus » expérimentés ( $n=13 ; 16-41$ années). Le premier groupe a généré au total 172 réponses $(38,2 \%)$, soit une moyenne de 5,4 réponses par clinicien et par vignette, alors que le second a produit 278 réponses $(61,8 \%)$, soit une moyenne de 5,3 réponses par clinicien et par vignette). Dans chacun de ces groupes, nous avons identifié les cinq moins expérimentés (45 ans, moyenne 4,8 ans) et les cinq plus expérimentés (30-41 ans, moyenne 28 ans) dans le but de voir si des différences pouvaient être observées. Les moins expérimentés ont généré au total 115 réponses $(25,5 \%)$ moyenne 5,8 réponses par clinicien et par vignette - et les plus expérimentés 130 réponses $(28,8 \%)$ moyenne 6,5 réponses par clinicien et par vignette.

En ce qui concerne les cadres conceptuels, 14 cadres conceptuels distincts ont été nommés par les moins expérimentés ( 1,8 par clinicien) alors que 27 cadres l'ont été par les plus expérimentés ( 2 par clinicien). À noter qu'un clinicien parmi le groupe des moins expérimentés (13 ans d'expérience) a généré à lui seul quatre cadres conceptuels distincts pour l'ensemble des vignettes, alors que deux cliniciens parmi le groupe des plus expérimentés (respectivement 30 et 38 ans d'expérience) ont chacun généré cinq et quatre cadres conceptuels distincts.
Les cadres conceptuels suivants ont uniquement été nommés par le groupe des moins expérimentés : le modèle systémique et celui l'entrevue centrée sur le patient.

Les cadres conceptuels suivants ont uniquement été nommés par le groupe des plus expérimentés :

- théories: les théories cognitives (lien avec les connaissances antérieures, schémas, transfert des connaissances).

- Meilleures pratiques : l'observation directe du résident aide à poser un diagnostic sur l'organisation de l'entrevue ; la lecture orientée par un cas aide à la rétention ; la lecture des textes de base avant celle des textes plus spécialisés aide à structurer les connaissances.

- Modèles : modèle d'entrevue (contenu et processus); modèle CanMEDS ; modèle d'empathie de Kohut ; modèle de la rétroaction constructive de Ende ; modèle de la pratique clinique réflexive de Schön.

- Typologie nosographique : DSM-IV (Manuel diagnostique et statistique des troubles mentaux).

Les cinq cliniciens les moins expérimentés (45 ans) ont produit quatre cadres (moyenne 0,8 par clinicien), alors que les cinq plus expérimentés (3041 ans) en ont généré huit (moyenne 1,6 par clinicien). Ces derniers ont été les seuls à nommer les quatre cadres conceptuels suivants: théories cognitives ; modèle d'entrevue (contenu et processus); modèle de la rétroaction constructive centrée sur l'étudiant ; modèle de la pratique réflexive.

Lors de l'analyse des données, nous avons, à titre exploratoire, comparé les réponses des femmes à celles des hommes, ainsi que celles des pédopsychiatres à celles de leurs collègues provenant de la psychiatrie adulte. Aucune différence n'a été constatée, ni sur le contenu des réponses, ni sur les cadres conceptuels les sous-tendant.

\section{Discussion}

Les réponses des cliniciens enseignants

Les quatre vignettes éducatives écrites que nous avons utilisées ont stimulé des réponses riches et variées (450 réponses, 85 sujets distincts). Ce constat a aussi été fait dans l'étude de Côté et Bordage ${ }^{[23]}$ ) au cours de laquelle six vignettes avaient été utilisées. Nous 
Tableau IV. Cadres conceptuels distincts rapportés par vignette.

\begin{tabular}{|c|c|}
\hline Meilleure pratique : & L'observation directe du résident aide à poser un Dx sur l'organisation de l'entrevue \\
\hline Modèles ou approches : & Structure d'entrevue du Collège Royal des médecins et chirurgiens du Canada \\
\hline & Entrevue (contenu et processus) \\
\hline & Systémique (thérapie familiale) \\
\hline & Cadre CanMeds \\
\hline & Psychodynamique (transfert/contre-transfert) \\
\hline Théorie & Théories cognitives (ex. : connaissances organisées en réseau, transfert des apprentissages) \\
\hline Vignette 2 (Raisonnement & ue - compétence « Expertise médicale ») \\
\hline Meilleures pratiques : & Faire sa démarche diagnostique à partir d'un ou de plusieurs cas \\
\hline Modèles ou approches : & Rétroaction constructive centrée sur l'étudiant \\
\hline & Entrevue centrée sur le patient \\
\hline & Entrevue (modèle de Shee) \\
\hline Théorie : & Théories cognitives \\
\hline Typologie nosographique & DSM-1V (Manuel diagnostique et statistique des troubles mentaux) \\
\hline Vignette 3 (Relation médec & itient - compétence « Communication») \\
\hline Modèles ou approches : & Alliance thérapeutique : en particulier l'empathie \\
\hline & Psychodynamique \\
\hline & Systémique \\
\hline & Empathie (modèle de Kohut) \\
\hline & Éthique-bienveillance \\
\hline Vignette 4 (Choix de resso & éducatives - compétence « Érudition») \\
\hline Meilleures pratiques : & Lire en fonction d'un cas \\
\hline & Lire les textes de base avant les textes plus spécialisés \\
\hline Modèles ou approches : & $\begin{array}{l}\text { Enseignement centré sur les besoins/difficultés de l'internet } \\
\text { Pratique réflexive (ex. : Schön) }\end{array}$ \\
\hline & Psychodynamique \\
\hline
\end{tabular}


retenons que ce choix méthodologique est pertinent et efficace car si on le compare, par exemple, à l'observation de discussions de cas tirés du contexte réel de pratique, l'élaboration de vignettes écrites permet de contrôler le contenu des demandes éducatives à partir duquel les cliniciens doivent répondre.

Une autre similitude avec l'étude de Côté et Bordage est que les réponses des cliniciens étaient spécifiques des cas (et des compétences) dans une proportion de $72 \%$. À l'instar de l'apprentissage du raisonnement clinique ${ }^{[30]}$, l'apprentissage de la rétroaction à l'étudiant doit avant tout reposer sur un répertoire varié d'interventions permettant de répondre spécifiquement aux demandes de l'étudiant et non seulement sur des principes pédagogiques ou cliniques généraux.

Par ailleurs, on remarque que, quelles que soient les vignettes, les cliniciens donnent généralement des réponses de type « conseils » (par exemple : conseils sur le choix de lecture, sur l'approche de traitement). Ils semblent poser moins de questions de clarification (par exemple : qu'as-tu trouvéà l'examen physique ?) et encore moins des questions d'approfondissement tel que le suggèrent Kennedy et Lindgard ${ }^{[31]}$ pour bien évaluer les compétences cliniques. De plus, il semble établi que, lors de la rétroaction, les questions d'approfondissement (probing-challenging) aident l'étudiant à atteindre un plus haut niveau d'analyse et de performance ${ }^{[8]}$. Il semble donc important d'inciter les cliniciens enseignants à développer leur répertoire de questions stimulantes, quel que soit le sujet discuté avec l'étudiant. Il est possible, par exemple, de demander au résident de s'autoévaluer avant le début de la rétroaction, de prioriser ses diagnostics différentiels et de les justifier ou encore de lui demander les stratégies qu'il envisage pour sortir d'une impasse relationnelle avec un patient. Les questions stimulantes sont une manière d'inciter l'étudiant à être actif dans ses apprentissages. À ce sujet, des suggestions de questions favorisant la pratique réflexive lors de la discussion de cas ont été récemment proposées par Ménard et Ratnapalan ${ }^{[32]}$.

Les cadres conceptuels

Les cliniciens enseignants ont explicitement rapporté plusieurs cadres conceptuels, dont 19 distincts au total. Tel qu'indiqué par Bordage ${ }^{[22]}$, le fait pour le clinicien enseignant d'avoir plusieurs types de cadres conceptuels en tête (théories, meilleures pratiques, modèles ou approches, paradigmes) peut l'aider à avoir une meilleure compréhension globale et spécifique de la situation, ainsi qu'un répertoire plus riche de réponses possibles à faire à l'interne. C'est un peu comme un menuisier qui possède un coffre contenant des outils diversifiés qu'il peut utiliser selon la nature du travail à réaliser.

Nous avons remarqué que certains cadres sont cliniques (par exemple : la méthode clinique centrée sur le patient) alors que d'autres sont pédagogiques (par exemple: les principes de la rétroaction constructive centrée sur l'étudiant), ces deux types de cadres visant des buts différents. Pour Ericsson et al. ${ }^{[8]}$, le fait d'avoir une pratique pédagogique diversifiée (mixed practice) fournit non seulement des alternatives permettant de répondre à des besoins pédagogiques variés des internes mais aide le superviseur à éviter, comme en clinique, les « fermetures prématurées » lorsqu'il explore les besoins pédagogiques de l'interne. Par contre, quelques cadres rapportés ne sont pas véritablement des cadres conceptuels au sens propre du terme. C'est ainsi que le «Manuel diagnostique et statistique des troubles mentaux », de même que le cadre CanMEDS, dont certains auteurs questionnent la nature ${ }^{[33]}$, s'apparentent davantage à de simples typologies, respectivement des entités nosographiques en psychiatrie ou des rôles professionnels en médecine.

Il est intéressant de constater que tout comme les réponses, les cadres étaient, dans l'ensemble, spécifiques des vignettes. Ce résultat concorde aussi avec l'étude de Côté et Bordage. Il apparaît donc que le choix des cadres semble habituellement être le fruit d'une démarche de raisonnement clinique et pédagogique prenant assise sur des informations présentées par l'interne ou sollicitées par le clinicien lors de la rétroaction. En ce sens, les cliniciens utiliseraient des scénarios pédagogiques -educational scripts- pour répondre aux besoins éducatifs des internes, un peu comme les scénarios cliniques mis en évidence par Irby il y a plusieurs années ${ }^{[34]}$.

En raison du nombre de participants ayant plusieurs années d'expérience, nous nous serions attendus 
à ce que les cliniciens enseignants expriment spontanément davantage de cadres, ce que plusieurs n'ont pu faire, malgré la méthode utilisée (rappel stimulé). Ainsi, pour les demandes pédagogiques en dehors du domaine de la démarche de raisonnement clinique, plusieurs ne savaient pas quoi répondre pour expliquer les réponses qu'ils avaient données dans la première partie de l'entrevue et ils ont souvent fait référence à leur expérience clinique ou pédagogique, sans pour autant être en mesure d'expliciter les bases de leurs réponses (par exemple : «Je fais ça comme ça et ça fonctionne mais je ne sais pas pourquoi »). Deux hypothèses pourraient expliquer ce phénomène : 1) l'absence de bases conceptuelles explicites ou la difficulté d'accès à ces bases ; 2) le manque de standardisation dans la manière dont la question a été posée aux cliniciens. En effet, même si les questions se rapportant à la deuxième partie de l'entrevue avaient été définies au départ, il s'avère que les questions qui ont été posées variaient parfois d'une entrevue à l'autre et pouvaient présenter une ambiguïté quant à l'objet recherché. Cela a pu créer de la confusion et ainsi limiter les réponses des précepteurs. Pour cette raison, les données sur la variété des cadres conceptuels des précepteurs demeurent partielles et devront être à nouveau explorées dans de futures études.

\section{L'influence de l'expérience}

Dans l'ensemble, les cliniciens plus expérimentés -et notamment les cinq les plus expérimentés- ont généré davantage de réponses et de cadres conceptuels que leurs collègues moins expérimentés. Pour autant, ce résultat ne permet pas d'affirmer que l'expérience influence le nombre de réponses et de cadres conceptuels des cliniciens. En effet, il est aussi possible que la richesse et l'étendue du répertoire des réponses soient dues à la présence d'une variable confondante qu'est la formation pédagogique reçue. Ainsi, les cliniciens plus expérimentés ont peut-être participé à davantage d'activités de formations pédagogiques, notamment sur la rétroaction et sur les principes d'apprentissage. Nous pouvons formuler l'hypothèse que les cliniciens ayant peu ou pas participé à des formations pédagogiques vont davantage reproduire le mode de supervision qu'ils ont eux-mêmes reçu sans pour autant être en mesure d'expliciter leurs stratégies pédagogiques. Lors de futures études, il faudra prendre ce facteur en considération. Par ailleurs, il faudra aussi beaucoup mieux définir le niveau d'expérience des participants car dans le groupe des moins expérimentés, on retrouvait notamment des cliniciens de niveau intermédiaire (par exemple, 13 ans d'expérience). Ainsi, au regard du critère du nombre d'années d'expérience, il pourrait s'avérer opportun de fixer respectivement les seuils d'inclusion, par exemple, à moins de trois années d'expérience clinique pour le statut «moins expérimenté » et à au moins 15 ans d'expérience pour le statut «plus expérimenté ».

Outre les limites mentionnées précédemment, cette étude aurait aussi pu inclure des vignettes mettant en évidence d'autres besoins éducatifs des internes (par exemple, la collaboration interprofessionnelle). De plus, nous aurions pu inclure des psychiatres provenant d'autres milieux afin de permettre une meilleure généralisation des résultats. Même si l'étude de Côté et Bordage visait à répondre sensiblement aux mêmes questions, les comparaisons entre notre étude et la leur doivent être faites avec prudence car les vignettes étaient moins nombreuses dans cette étude-ci. De plus, alors que l'étude de Côté et Bordage a établi une corrélation positive entre le nombre de réponses et le nombre de cadres conceptuels, notre étude ne s'est pas intéressée à cette question et une prochaine étude devra s'y attacher. Dans le futur, nous pourrions aussi réaliser une étude observationnelle des cliniciens en situation de discussion de cas avec leurs internes, afin d'analyser les réponses qu'ils donnent, en contexte réel, et ce que les internes retiennent.

Malgré ses limites, cette étude qualitative satisfait de façon acceptable les critères de rigueur scientifique reconnus pour ce type d'étude, soit la crédibilité et la transférabilité. En ce qui concerne la crédibilité, nous nous sommes notamment préoccupés de la diversité des participants, de la saturation des données et de la triangulation des chercheurs. Quant à la transférabilité des données, nous souscrivons aux propos de Kuper et al. ${ }^{[35]}$ qui indiquent que les résultats des études qualitatives ne peuvent ni ne doivent être généralisés de la même manière que les études 
quantitatives. Les résultats sont transférables à d'autres contextes, dans la mesure où les lecteurs jugent que les caractéristiques des contextes et des participants des études sont comparables à celles de leurs propres contextes.

\section{Conclusion}

Cette étude est la première dédiée à cette thématique en psychiatrie. Elle a permis de documenter les réponses des cliniciens enseignants à partir de vignettes standardisées, mettant en évidence des demandes éducatives lors de présentations de cas faites par les résidents, ainsi que les bases conceptuelles sur lesquelles reposent ces réponses. Dans une optique de transfert des connaissances, nos résultats seront très utiles pour l'élaboration d'activités de formation pédagogique sur le contenu de la rétroaction en contexte clinique et sur les fondements s'y rapportant. De plus, les résultats de nos travaux, combinés à ceux de Côté et Bordage fournissent plusieurs pistes de recherche prometteuses.

\section{Contributions}

Luc Côté et Nathalie Gingras ont participé à toutes les étapes de la conception et de la réalisation de l'étude, ainsi qu'à celles de la rédaction et de la révision de cette publication.

\section{Remerciements}

Nous tenons à remercier les cliniciens enseignants en psychiatrie associés au réseau d'enseignement de l'Université Laval, qui ont participé à cette étude, ainsi que le Fonds Gilles-Cormier de la Faculté de médecine de l'Université Laval qui, par son soutien financier, a permis la réalisation de cette recherche. De plus, nous remercions Marie-Ève Riopel pour sa contribution à la réalisation des entrevues, ainsi que Jean-François Roux pour ses commentaires et suggestions se rapportant à une version antérieure du manuscrit.

\section{Approbation éthique}

L'approbation préalable du protocole de recherche a été obtenue par le Comité d'éthique de la recherche de l'Université Laval. Cette étude nécessitait l'approbation du CÉRUL et nous l'avons obtenue. Le consentement éclairé de tous les participants a été obtenu par écrit. Les transcriptions ont été anonymisées.

\section{Déclaration d'intérêts}

Les auteurs ne signalent aucun conflit d'intérêt en lien avec le contenu de cet article.

\section{Références}

1. Kilminster SM, Jolly BC. Effective supervision in clinical settings: a literature review. Med Educ 2000;34:827-40.

2. Dolmans DHJM, Wolfhagen HAP, Gerver WJ, De Grave W, Scherpbier AJJA. Providing physicians with feedback on how they supervise students during patient contacts. Med Teach 2004;26:409-14.

3. Dornan T, Littlewood S, Margolis SA, Scherpbier A, Spencer J, Ypinazar V. BEME GUIDE 6: How can experience in clinical and community settings contribute to early medical education? A BEME systematic review. Med Teach 2006;28:3-18.

4. Kilminster SM, Cottrell D, Grant J, Joly BC. AMEE Guide no. 27: Effective educational and clinical supervision. Med Teach 2007;29:2-19.

5. Saucier D, Paré L, Côté L, Baillargeon L. How core competencies are taught during clinical supervision: participatory action research in family medicine. Med Educ 2012;46:1194-205.

6. Wenger E, McDermott R, Snyder W. Cultivating communities of practice. A guide to managing knowledge. Boston: Harvard Business School Press, 2002.

7. Ericsson KA, Deliberate practice and the acquisition and maintenance of expert performance in medicine and related domains. Acad Med 2004;79:S70-S81.

8. Ericsson KA, Charness N, Feltovich PJ, Hoffman RR (Eds.). The Cambridge handbook of expertise and 
expert performance. New York: Cambridge University Press, 2006.

9. Connell KJ, Bordage G, Chang RW, Howard BA, Sinacore J. Measuring promotion of thinking during precepting encounters in outpatient settings. Acad Med 1999;74:S10-S12.

10. Bowen JL, Irby DM. Assessing quality and costs of education in the ambulatory setting: a review of the literature. Acad Med 2002;77:621-80.

11. Jouquan J. Le raisonnement clinique et la supervision pédagogique sous l'éclairage des métaphores et des analogies. Pédagogie Médicale 2013;14:79-81.

12. Collins A, Brown JS, Newman SE. Cognitive apprenticeship: teaching the crafts of reading, writing, and mathematics. In: Resnick LB (ed). Knowing, learning, and instruction: Essays in honor of Robert Glaser. Hillsdale (NJ): Lawrence Erlbaum Associates, 1989.

13. van de Ridder JMM, Stokking KM, McGaghie, WC, ten Cate OTJ. What is feedback in clinical education? Med Educ 2008;42:189-97.

14. Archer JC. State of the science in health professional education: effective feedback. Med Educ 2010;44:10108.

15. Neher JO, Gordon KC, Meyer B, Stevens N. A fivestep "microskills" model of clinical teaching. J Am Board Fam Pract 1992;5:419-24.

16. Wolpaw T, Côté L, Papp K, Bordage G. Student uncertainties drive teaching during case presentations: More so with SNAPPS. Acad Med 2012;87:1210-17.

17. Dewey J. How We Think. Boston: Heath, 1933.

18. Joplin L. On defining experiential education. Journal of Experiential Education 1981;4:17-20.

19. Schön DA. The reflective practitioner: how professionals think in action. New York: Basic Books, 1983.

20. Eva KW, Regehr G. Self-assessment in the health professions: A reformulation and research agenda. Acad Med 2005;80:S46-S54.

21. Giroux M, Girard G. Favoriser la position d'apprentissage grâce à l'interaction superviseur-supervisé. Pédagogie Médicale 2009;10:193-210.

22. Bordage G. Conceptual frameworks to illuminate and magnify. Med Educ 2009;43:312-19.

23. Côté L, Bordage G. Content and conceptual frameworks of preceptor feedback related to residents' educational needs. Acad Med 2012;87:1274-81.

24. Côté L, Turgeon J. Appraising qualitative research articles in medicine and medical education. Med Teach 2005;27:71-5.
25. Pelaccia T, Paillé P. Les approches qualitatives : une invitation à l'innovation et à la découverte dans le champ de la recherche en pédagogie des sciences de la santé. Pédagogie Médicale 2010;10:293-304.

26. Frank JR. (Réd.). Le Cadre de compétences CanMEDS 2005 pour les médecins. L'excellence des normes, des médecins et des soins. Ottawa : Le Collège royal des médecins et chirurgiens du Canada, 2005 [Online] Disponible sur : http://crmcc.medical.org/canmeds/CanMEDS2005/index.php

27. Fonteyn ME, Kuipers B, Grobe SJ. A description of think aloud method and protocol analysis. Qual Health Res 1993;3:430-41.

28. Lyle J. Stimulated recall: a report on its use in naturalistic research. Br Educ Res J 2003;29:861-78.

29. L'Ecuyer R. L'analyse de contenu : notions et étapes. In : Deslauriers JP. Les méthodes de la recherche qualitative. Sillery: Presses Universitaires du Québec, 1988:49-65.

30. Nendaz M, Charlin B, Leblanc V, Bordage G. Le raisonnement clinique : données issues de la recherche et implications pour l'enseignement. Pédagogie Médicale 2005;6:235-54.

31. Kennedy TJT, Lingard LA. Questioning competence: A discourse analysis of attending physicians' use of questions to assess trainee competence. Acad Med 2007;82:S12-S15.

32. Ménard L, Ratnapalan S. Réflexion en médecine. Modèles et application. Can Fam Physician 2013;59:e57-9.

33. Parent F, Jouquan J, De Ketele JM. CanMEDS and other "competency and outcome-based approaches" in medical education: clarifying the ongoing ambiguity. Adv Health Sci Educ Theory Pract 2013; 18:115-22

34. Irby DM. How attending physicians make instructional decisions when conducting teaching rounds. Acad Med 1992;67:630-38.

35. Kuper A, Reeves S, Levinson W. An introduction to reading and appraising qualitative research. BMJ 2008;337:404-7.

Correspondance et offprints : L. Côté, Département de médecine familiale et de médecine d'urgence. Pavillon Ferdinand-Vandry, bureau 2881-E. Université Laval 1050, avenue de la Médecine, G1V 0A6 Québec, Canada.

Mailto : luc.cote@fmed.ulaval.ca 
Annexe 1. Réponses regroupées par catégories.

\section{Compétence « Érudition » (Vignette 4)}

Exploration du vécu / support au résident

- Explorer le vécu du résident : problématique pédagogique vécue

• Exprimer au résident son soutien : problématique pédagogique vécue (ex. : normaliser) 13

- Valoriser le résident qui exprime une difficulté

- Transmettre une rétroaction qui est constructive pour le résident (ex. : bon dosage) 5

- Donner des conseils pratiques : bien-être du résident (ex. : se reposer) 2

Stratégies pour poser un diagnostic pédagogique

- Poser un diagnostic pédagogique provisoire ou définitif (ex. : problème de raisonnement clinique)

- Aider le résident à préciser son diagnostic pédagogique (ex. : en le questionnant sur sa démarche d'entrevue)13

- Proposer l'observation directe comme moyen de poser un diagnostic pédagogique

- Demander au résident de poser son propre diagnostic pédagogique

- Poser un diagnostic clinique du résident (ex. : résident qui a un trouble obsessionnel compulsif)

- Demander au résident de décrire sa démarche d'entrevue avec le patient

- Revoir le résident pour faire le suivi pédagogique

- Souligner l'importance de saisir les indices du patient pendant l'entrevue

- Utiliser la technique de l'incident critique comme moyen pour aider le résident à préciser ses forces et difficultés

- Questionner le résident sur ses connaissances des techniques d'entrevue

- Suggérer des lectures (ex. : textbook) ou ressources documentaires internet (ex. : Medline)

- Proposer un moyen pour chercher l'information (ex. : faire Medline ensemble

- Explorer les ressources d'information utilisées

- Explorer la démarche de recherche d'information du résident

- Inciter le résident à faire sa démarche de recherche d'information puis la comparer avec celle du patron

- Encourager le résident à transférer ses apprentissages

(ex. : lecture qui sera aussi utile à la préparation d'une future présentation)

- Vérifier que le résident a intégré l'information recherchée en réponse à ses questions

- Lire en fonction de différents axes selon la problématique du patient

- Vérifier les ressources de lecture auprès de collègues

- Mettre en garde contre le fait de trop lire / lire de manière non structurée

Apprenant à vie

-Souligner l'importance d'être un apprenant à vie 2

Stratégies pédagogiques générales $\quad 30$

- Revoir le patient avec le résident $\quad 7$

- Clarifier la demande pédagogique du résident 5

- Utiliser différentes stratégies pour amener le résident à considérer différentes dimensions du diagnostic (ex. : remettre en question les dogmes, questionner sur certains aspects auxquels il n'a pas pensé) 
- Proposer au résident de filmer son entrevue, de la revoir et d'en discuter avec son superviseur

- Rediscuter du cas avec le résident

- Travailler à partir de la compréhension que le résident a de la problématique,

dans une perspective de co-construction

2

- Identifier les difficultés du résident et lui demander sur quel aspect il veut travailler

2

- Préparer l'entrevue avec le résident

- Identifier les difficultés / défis potentiels de l'entrevue

- Faire un jeu de rôle de démonstration (patron jour le rôle du résident et résident joue le rôle du patient)

- Clarifier les décisions du résident (ex. : quelle est l'intention thérapeutique)

- Mettre le résident dans un rôle d'enseignant (ex. : conseils à un autre résident dans la même situation)

Compétence « Expertise médicale » (Vignette 2)

$\underline{\text { Histoire }}$

- Demander au résident des précisions sur la problématique clinique, faire raconter l'histoire par le résident

- Souligner l'importance d'explorer certains aspects de la problématique clinique

(ex. : résistance, contexte de vie)

- Souligner l'importance de faire une bonne histoire (ou questionnaire)

- Aller vérifier /compléter l'histoire auprès du patient afin de clarifier la problématique clinique (en présence du résident)

$\underline{\text { Connaissances }}$

- Vérifier les connaissances factuelles du résident

$\underline{\text { Raisonnement clinique }}$

- Donner des moyens concrets pour trouver les diagnostics différentiel et principal

(ex. : arbre décisionnel, tableau, carte conceptuelle, caractéristiques-clé du cas)

- Donner des moyens concrets comprendre la problématique du patient

- (ex. : travailler à partir de plusieurs cas, revoir le patient)

- Demander au résident de comparer/contraster les diagnostics

- Souligner l'importance de tolérer l'incertitude

- Souligner l'importance d'avoir une représentation globale du problème

- Souligner l'importance de relire sur les critères diagnostiqués

- Demander au résident sa première impression diagnostiqué (pattern recognition)

- Demander au résident sa compréhension du symptôme/tableau clinique

- Mettre en garde contre fermeture prématurée

- Souligner l'importance de considérer tous les diagnostics probables

- Souligner l'importance de poser le bon diagnostic

Évolution du problème

- Souligner l'importance d'objectiver l'évolution du problème

$\underline{\text { Suivi }}$

- Donner des conseils pour le suivi du patient (ex. : référence à une ressource appropriée) 
Sécurité du pt

- Souligner l'importance de vérifier la sécurité pour le patient (ex. : idées suicidaires)

Traitement /Conduite

- Donner des conseils sur l'approche de traitement (psychosocial, pharmaco)

- Questionner le résident sur les options thérapeutiques

- Mettre en garde contre certaines interventions (ex. : ne pas prescrire trop vite)

$\underline{\text { Gestion d'entrevue }}$

- Conseiller : techniques d'entrevues (ex. : se donner des repaires dans le temps ;

doser les questions ouvertes et fermées; reformuler)

- Conseiller : gestion d'un cas (ex. : établir un contrat thérapeutique avec le patient, revoir le patient, bien connaître son champs d'expertise)

- Proposer un cadre/démarche : structure d'entrevue (ex. : en fonction des objectifs de l'entrevue)

- Préciser l'attente face au temps d'entrevue tout en nuançant son application

(ex. : cadre des examens du CRMCC)

- Explorer auprès du résident ses solutions antérieures : même type de cas

- Donner des conseils : compréhension commune

- Prendre plus de temps avec le patient pour poser le diagnostic

Compétence « Communication » (Vignette 3)

Alliance thérapeutique

- Donner des conseils pour favoriser l'alliance thérapeutique (ex. : démontrer empathie)

- Souligner l'importance d'explorer le lien thérapeutique patient-médecin antérieur

- Souligner l'importance d'établir une bonne alliance thérapeutique comme condition d'une bonne relation médecin-patient

- Aider le résident à approfondir la compréhension de ce qui se passe avec le patient (ex. : relation contre-transférentielle)

- Souligner l'importance d'explorer le vécu du patient

- Mettre en garde d'ajuster /adapter ses messages au patient pour établir/consolider

l'alliance thérapeutique

- Démontrer comment faire via le modèle de rôle

- Souligner l'importance d'explorer l'alliance avec le patient

$\underline{\text { Adhésion au traitement }}$

- Donner des conseils pour favoriser l'observance du patient

- Souligner l'importance d'explorer avec le patient les raisons de la non-observance

- Souligner l'importance d'explorer la compréhension du patient : problématique

- Aider le résident à tolérer la résistance du patient

- Souligner l'importance de vérifier l'observance du patient (ex. : médication)

Implication de la personne accompagnatrice

- Donner des conseils afin d'impliquer la personne accompagnatrice (ex. : solliciter son point de vue)

- Souligner l'importance d'explorer le vécu /impact de la personne accompagnatrice 


\section{Compétence Gestion (Vignette 1)}

Importance de gérer les soins

- Conseiller : gestion de l'horaire (ex. : alterner cas évaluation et suivi, rédaction du dossier)

- Souligner l'importance de gérer la charge de cas

- Souligner l'importance d'un équilibre vie personnelle-professionnelle

Annexe 2. Vignettes utilisées lors des entretiens avec les cliniciens.

\section{Vignette 1 : « J'aimerais bien être plus efficace » (Compétence « Gestion »)}

Je viens de voir un nouveau patient; heureusement que c'est le dernier de ma journée. Il s'agit de Monsieur Levasseur, 50 ans qui consulte aujourd'hui pour évaluation d'un trouble anxieux. Il est ingénieur en informatique. En plus de son problème d'anxiété, il a plusieurs problèmes de santé. Je suis finalement passé au travers du questionnaire avec ce nouveau patient. Je ne peux pas croire tout le temps que ça m'a pris. En faisant le questionnaire, il me répondait avec tellement de détails que ça n’en finissait plus. Au moment où je me préparais à venir vous parler, il m'a aussi dit qu'il était inquiet d'avoir une maladie sexuellement transmissible parce qu'il avait eu une relation non protégée avec une femme qu'il avait rencontrée dans un bar il y a 1 mois. Il a consulté son médecin de famille et il est en attente des résultats. Il est surtout anxieux à l'idée de devoir en parler à sa femme. À la fin, j'ai dit à Monsieur Levasseur qu'il fallait vraiment que je vienne discuter avec vous. C'est mon dernier rendez-vous aujourd'hui et comme vous pouvez le constater, je suis en retard, ce qui a tendance à m'arriver souvent. Je suis toujours le dernier à partir en fin de journée. J'aimerais bien être plus efficace.

\section{Vignette 2 : « Je n’arrive pas à me faire une idée » (Compétence « Expertise médicale »)}

Je viens de voir Monsieur Martin âgé de 34 ans que j'avais vu il y a 15 jours et qui revient aujourd'hui pour un suivi. Il consultait pour des idées suicidaires qui ont débutées il y a environ 1 1/2 mois. Au début, je pensais qu'il présentait une dépression car je notais comme symptôme de la tristesse, des idées suicidaires et de la fatigue. Je me demandais aussi si ça pouvait être un trouble d'anxiété généralisé car c'est un monsieur anxieux de nature et qui anticipe toujours les situations. Aujourd'hui, il me parle aussi de stress au travail et je me demandais s'il peut avoir plutôt un trouble d'adaptation. En fait, plus je parle de ce patient-là, moins c'est clair pour moi la différence entre la dépression, le trouble d'adaptation et le trouble anxieux généralisé. J'ai lu pas mal sur ces pathologies mais ici, je tourne en rond. Je n'arrive pas à me fixer sur un diagnostic. Malgré toutes mes connaissances sur le sujet, je n'arrive pas à me faire une idée.

\section{Vignette 3 : «Avez-vous des trucs à me donner ? » (Compétence « Communication »)}

Je voudrais vous parler de Mme Tremblay. Elle a 19 ans et a un diagnostic de trouble psychotique. Je viens de la voir avec sa mère. Je suis le nouveau résident qui s'occupe d'elle, le précédent ayant terminé sa résidence. C'était la Dre Smith. À chaque fois que je vois Mme Tremblay, elle me parle de cette résidente et de combien elle est triste de son départ. C'est clair qu'elle n'a pas fait son deuil. En tout cas, avant aujourd'hui, j'ai vu Mme Tremblay une seule fois et c'était il y a 2 mois. En plus de son problème psychotique, elle a pris du poids avec la médication et présente un début de syndrome métabolique. Ses glycémies étaient élevées ; je l'ai référée à une diététiste et faite évaluer en endocrinologie. Aujourd'hui, je note que son poids a encore augmenté. J'ai regardé avec elle ce qui 
s'était passé depuis le dernier rendez-vous pour me rendre compte qu'elle n'avait pas suivi l'entente qu'on avait prise la dernière fois : elle devait surveiller sa diète et prendre 2-3 marches par semaine. La dernière fois, $\mathrm{j}$ 'avais pris la peine de bien vérifier si elle était d'accord avec ça. Aujourd'hui elle me dit que pour les marches, elle n'avait pas eu le temps et pour la diète, qu'elle n'y avait pas pensé. J'ai dit à Madame Tremblay que si elle ne collaborait pas, c'est certain que ses problèmes de santé s'aggraveraient. Je lui ai dit qu'elle pourrait peut-être avoir des problèmes cardiaques et d'autres complications sérieuses si elle ne respectait pas les recommandations. Elle s'est mise à pleurer et sa mère s'est fâchée et m'a dit qu'elle trouvait inacceptable que je fasse pleurer sa fille. J'avoue que je ne sais plus trop quoi faire. Auriez-vous des trucs à me donner avec ce genre de situation-là ?

\section{Vignette 4 : «Qu'est-ce que je pourrais lire ? » (Compétence « Érudition »)}

Est-ce que vous pourriez m'aider? J'ai un patient, Monsieur Juneau, âgé de 45 ans, qui a un trouble obsessionnel compulsif depuis plusieurs années. C'est mon premier cas de trouble obsessionnel compulsif et en plus, il semble réfractaire aux traitements habituels. J'aimerais lire sur le sujet afin d'être mieux préparé pour le rencontrer. Que me conseillez-vous? 